\title{
Reflectance confocal microscopy- guided laser ablation of basal cell carcinomas: initial clinical experience
}

\author{
Heidy Sierra \\ Oriol Yélamos \\ Miguel Cordova \\ Chih-Shan Jason Chen \\ Milind Rajadhyaksha
}




\title{
Reflectance confocal microscopy-guided laser ablation of basal cell carcinomas: initial clinical experience
}

\author{
Heidy Sierra, ${ }^{\mathrm{a}, *, \dagger}$ Oriol Yélamos, ${ }^{\mathrm{a}, \mathrm{b}}$ Miguel Cordova, ${ }^{\mathrm{a}}$ Chih-Shan Jason Chen, ${ }^{\mathrm{a}, \neq}$ and Milind Rajadhyaksha ${ }^{\mathrm{a}, \neq}$ \\ ${ }^{a}$ Memorial Sloan Kettering Cancer Center, Dermatology Service, New York, New York, United States \\ bUniversitat de Barcelona, Hospital Clínic, Dermatology Department, Barcelona, Spain
}

\begin{abstract}
Laser ablation offers a procedure for precise, fast, and minimally invasive removal of superficial and early nodular basal cell carcinomas (BCCs). However, the lack of histopathological confirmation has been a limitation toward widespread use in the clinic. A reflectance confocal microscopy (RCM) imaging-guided approach offers cellular-level histopathology-like feedback directly on the patient, which may then guide and help improve the efficacy of the ablation procedure. Following an ex vivo benchtop study (reported in our earlier papers), we performed an initial study on 44 BCCs on 21 patients in vivo, using a pulsed erbium:ytterbium aluminum garnet laser and a contrast agent (aluminum chloride). In 10 lesions on six patients, the RCM imagingguided detection of either presence of residual tumor or complete clearance was immediately confirmed with histopathology. Additionally, 34 BCCs on 15 patients were treated with RCM imaging-guided laser ablation, with immediate confirmation for clearance of tumor (no histopathology), followed by longer-term monitoring, currently in progress, with follow-up imaging (again, no histopathology) at 3, 6, and 18 months. Thus far, the imaging resolution appears to be sufficient and consistent for monitoring efficacy of ablation in the wound, both immediately postablation and subsequently during recovery. The efficacy results appear to be promising, with observed clearance in 19 cases of 22 cases with follow-ups ranging from 6 to 21 months. An additional 12 cases with 1 to 3 months of follow-ups has shown clearance of tumor but a longer follow-up time is required to establish conclusive results. Further instrumentation development will be necessary to cover larger areas with a more automatically controlled instrument for more uniform, faster, and deeper imaging of margins. ๑ 2017 Society of Photo-Optical Instrumentation Engineers (SPIE) [DOI: 10.1117/1.JBO.22.8.085005]
\end{abstract}

Keywords: image-guided therapy; laser ablation; confocal microscopy; skin cancer; basal cell carcinoma.

Paper 170228PR received Apr. 11, 2017; accepted for publication Jul. 26, 2017; published online Aug. 23, 2017.

\section{Introduction}

Basal cell carcinoma (BCC) is one of the most common types of cancer prevalent around the world. ${ }^{1,2}$ BCCs appear mostly on the face and head-and-neck areas. Mohs surgery is one of the standard procedures to precisely remove BCCs, while minimizing damage to the surrounding normal tissue. ${ }^{3}$ Mohs surgery is guided by the preparation and examination of histopathology, performed in parallel during surgery, which makes the process highly effective with high cure rates, but labor intensive, time consuming, and high cost.

Nearly 600,000 superficial and early nodular types of BCCs are currently treated in the USA with Mohs surgery every year, accounting for an estimated $30 \%$ to $40 \%$ of cases. Because these are superficial (within $\sim 200-\mu \mathrm{m}$ depth), low-risk, nonaggressive, nonmetastatic, slow-growing, and nonfatal cancers, they are being increasingly treated with alternative less invasive, with some being potentially lower cost, treatments, such as curettage and electrodessication, topical drug therapy, photodynamic therapy, radiotherapy, and laser therapy. ${ }^{2,4}$ Laser therapies, in the form of ablation, offer relatively quick, highly localized, and precise removal of superficial and early nodular

\footnotetext{
*Address all correspondence to: Heidy Sierra, E-mail: heidy.sierra1 @upr.edu ${ }^{\dagger}$ Current address: University of Puerto Rico Mayaguez, Department of Computer Science and Engineering, Mayaguez, Puerto Rico, United States.

¥The authors contributed equally to this work.
}

BCCs. Laser therapy with carbon dioxide $\left(\mathrm{CO}_{2}\right)$, pulsed dye, erbium:yttrium aluminum garnet (Er:YAG), and neodymium: yttrium aluminum garnet $(\mathrm{Nd}: \mathrm{YAG})$ has been explored for treatment of BCCs. ${ }^{5-18}$

However, laser therapy is yet to become a widely used treatment because the skin is either vaporized or coagulated, and there is no tissue available for immediate histopathological confirmation of clearance of tumor. Consequently, the resulting efficacy and cure rate have been variable, and thus a major barrier against further advances and more widespread use. ${ }^{10}$ However, with new advances in high-resolution optical and ultrasound imaging technology, image-guided laser therapy seems to be a possible logical solution to address these obstacles. Instead of histopathology, imaging may be performed directly on patients, to confirm clearance and monitor long-term efficacy. Initial clinical studies appear to be promising. ${ }^{17,18}$

In a previous benchtop study on skin tissue ex vivo, we demonstrated that high-resolution nuclear-level reflectance confocal microscopy (RCM) imaging can detect the presence of residual tumor or clearance in postablated skin tissue. ${ }^{19,20}$ Ablation was performed using an Er:YAG laser $(2900 \mathrm{~nm})$ with a pulse duration of $250 \mu \mathrm{s}$. The depth of ablation was characterized as a function of fluence (range 6.3 to $25.0 \mathrm{~J} / \mathrm{cm}^{2}$ ) and number of passes (range 1 to 6 , one pass was equal to four pulses). The results showed that a single treatment of 1 to 6 passes, each

1083-3668/2017/\$25.00 @ 2017 SPIE 
with a fluence of $25 \mathrm{~J} / \mathrm{cm}^{2}$ (i.e., maximum total fluence of $150 \mathrm{~J} / \mathrm{cm}^{2}$ ) allows removal of BCCs, with very minimal thermal coagulation on the wound surface and the surrounding intact (untreated) tissue. Consequently, RCM imaging, with a topically applied contrast agent to enhance nuclear contrast, can be then used to detect the presence or absence of tumor postablation. Results also showed that, by introducing $\sim 2 \mathrm{~s}$ of waiting time after six passes to allow for tissue cooling, further treatment with more passes could be performed, and thermal coagulation can be well controlled to allow for high-quality imaging postablation.

Following the benchtop study, we proceeded to initial testing in two patients, ${ }^{18}$ as the first baby step in the surgical setting, toward a subsequent larger study. The testing showed the feasibility of RCM imaging to provide immediate histopathologylike feedback for detection of either residual BCCs or clearance directly on the patient after laser ablation. Aluminum chloride (routinely used for hemostasis after skin biopsies or excisions) was used as a contrast agent, topically applied on the surface of the postablated wound to enhance contrast of nuclear patterns and tumor morphology. (In an early imaging study, aluminum chloride was serendipitously discovered to brighten nuclei and behave as a contrast agent for BCCs. ${ }^{21}$ ) Confirmation of the imaging findings with histopathology showed RCM imaging quality and performance similar to that observed in the earlier ex vivo studies.

Building upon our ex vivo study and initial in vivo testing, we then developed an RCM imaging-guided laser ablation protocol for larger-scale implementation and testing. In this paper, we present a detailed description of the protocol and our initial experience in the implementation on patients. The protocol has been tested in 44 lesions on 21 patients, each diagnosed as nodular and/or superficial BCC. The protocol consists of preablation detection of lateral and deep margins of BCCs, which then guides the choice of ablation parameters (fluence, number of pulses), followed by laser ablation, and, finally, imaging of the peripheral epidermis and the deeper dermis in the wound bed to provide feedback on the presence of residual tumor or clearance. In the initial 10 lesions, for which diagnostic biopsy was available, the postablation RCM findings were immediately confirmed with a thin excision and histopathology. The subsequent 34 lesions, which were clinically diagnosed [i.e., with only clinical (visual) examination and dermoscopy but without diagnostic biopsy], were treated with imaging-guided laser ablation, followed by immediate confirmation of clearance of tumor (no histopathology), and further longer-term monitoring, currently in progress, with follow-up imaging (again, no histopathology). We present the imaging performance and results for both the initial 10 lesions and the subsequent 34 lesions, and discuss the current limitations of this approach and possible solutions for further advances.

\section{Materials and Methods}

\subsection{Laser Ablation Instrumentation}

A pulsed erbium-doped yttrium aluminum garnet (Er:YAG, $2.94 \mu \mathrm{m}$ ) laser (Sciton Profile, Palo Alto, California), equipped with a pulse duration of $\sim 250 \mu$ s (fixed), spot diameter of $4 \mathrm{~mm}$, and fluence up to $25.0 \mathrm{~J} / \mathrm{cm}^{2}$ was used. More details can be found in our earlier papers. ${ }^{19,20}$ One "pass" of $\sim 161$ ms duration consists of a set of four individual pulses, each separated by $40 \mathrm{~ms}$. [Note that shorter pulse durations, relative to the thermal relaxation time in skin, are usually preferable, such as femtosecond or picosecond lasers, which would result in highly selective "pure" ablation of thin cellular-level layers of tissue without any underlying thermal coagulation. Our particular choice of laser and pulse duration was mainly due to the routine use of Er: YAG lasers in dermatologic clinical settings, and thus the easy availability and access to the laser used by our collaborating Mohs surgeon (coauthor CSJC). For ablating superficial or nodular BCCs of $\sim 150$ - to $200-\mu \mathrm{m}$ depth, very high selectivity is not necessary, and longer pulse durations on the order of 10 to 100 microseconds are clinically sufficient.] In this study, the fluence was fixed at $25.0 \mathrm{~J} / \mathrm{cm}^{2}$ for a single pass and, when necessary, a higher fluence was obtained by performing several passes, to produce a depth of ablation that reached the tumor's deepest margins. The selection of the number of passes was based on an ex vivo study in human skin specimens, previously conducted, to characterize the depth of ablation versus number of passes. Increasing the number of passes to produce higher fluence values also increases the thermal residual damage to surrounding intact tissue postablation. ${ }^{22}$ As reported in our previous benchtop studies, the thermal damage depth for these laser parameters is 5 to $20 \mu \mathrm{m}$, confirming the literature. ${ }^{22}$ Our study provided a set of parameters (number of passes at fixed fluence) to ablate BCCs while controlling thermal coagulation in the underlying wound and surrounding intact skin, to subsequently allow the uptake of contrast agent and enhance the appearance of nuclear patterns and tumor morphology for the detection of presence (or absence) of tumor postablation with RCM imaging. Thus, the uptake of aluminum chloride (seen in this in vivo study as well as in the previous benchtop studies), along with the wound healing and recovery process, indicates that healthy tissue remains undamaged. Further details may be gleaned from our earlier papers. ${ }^{19,20}$

\subsection{Patients and Basal Cell Carcinomas Lesions}

Twenty-one patients, with forty-four lesions, each with a diagnosis of superficial and/or nodular BCC, participated in this study. Prior to enrollment, patients gave consent to a research protocol, approved by Memorial Sloan Kettering Cancer Center's (MSKCC's) Institutional Review Board, for imaging and ablation. An initial group of six patients, with 10 BCCs that were confirmed with diagnostic biopsy, consented for RCM imaging-guided laser ablation. On these patients, immediately postablation, a thin excision was performed and the resulting frozen histology used for confirmation. A second set of 15 patients, on whom 34 superficial and early nodular BCCs had been clinically diagnosed [i.e., with only clinical (visual) examination and dermoscopy but without diagnostic biopsy], was treated with RCM imaging-guided laser ablation only (i.e., with no subsequent excision or histopathology), and all of these lesions are currently being followed up and noninvasively monitored with imaging.

\subsection{Reflectance Confocal Microscopy Imaging}

Imaging on patients was performed with two reflectance confocal microscopes [Vivascope 1500 and Vivascope 3000, Caliber Imaging and Diagnostics (formerly, Lucid Inc.), Rochester, New York]. In both, the illumination is with the near-infrared wavelength of $830 \mathrm{~nm}$, and imaging was done with a gel-immersion objective lens of magnification $30 \times$ and 0.9 numerical aperture, optical sectioning of $\sim 3 \mu \mathrm{m}$ and lateral resolution of 
$\sim 1 \mu \mathrm{m}$. High-resolution RCM images were collected to a depth of $\sim 150$ to $200 \mu \mathrm{m}$.

\subsubsection{Preablation reflectance confocal microscopy imaging}

Preablation imaging of intact skin was performed with a larger version of the microscope (VivaScope 1500) through a tissue contact ring and polycarbonate window, which sits directly in contact with the skin to stabilize the area to be imaged. Oil is applied to the skin to match the refractive indices of both the window and the topmost stratum corneum layer of skin. The microscope allows the acquisition of single images and stacks of images with a field of view of $500 \mu \mathrm{m} \times 500 \mu \mathrm{m}$. By the use of an automated stepper that rasters the objective lens in two dimensions (2-D), a 2-D matrix of contiguous images can be captured and processed to create mosaics to increase the field of view to up to $8 \mathrm{~mm} \times 8 \mathrm{~mm}$. The mosaics of images can be captured and generated in $\sim 2 \mathrm{~min}$. Further details are below and more may be also gleaned from our earlier papers. ${ }^{23}$

\subsubsection{Postablation reflectance confocal microscopy imaging}

The surface of the wound was imaged using a smaller handheld version of the microscope (Vivascope 3000), with a small and integrated lens-to-skin tissue contact cap and polycarbonate window. While gently pressing the contact cap and window against the tissue and manually moving the microscope over the wound surface, images and/or videos are captured while covering a larger field of view. The videos are processed into video mosaics to show larger areas of tissue, in long strips of up to $\sim 1 \mathrm{~mm} \times \sim 30 \mathrm{~mm}$, to show the peripheral epidermal margin and deeper dermal margins (base of the wound).

Further details on the video mosaicking approach are below and more may also be gleaned from an earlier paper. ${ }^{24}$

\subsection{Image-Guided Laser Ablation Protocol}

Figures 1-3 show the complete perioperative imagingguided protocol, with histopathology-like feedback obtained
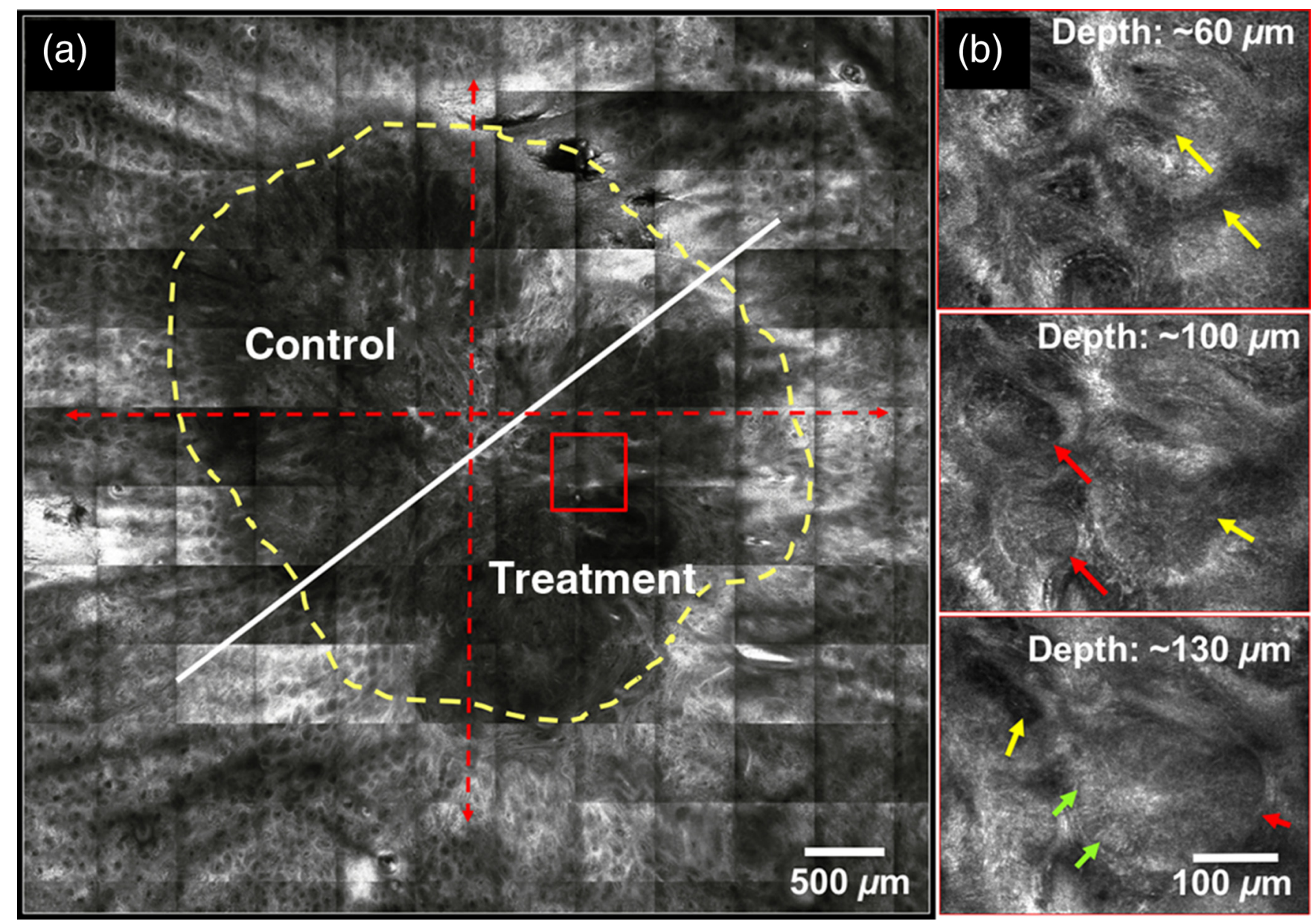

Fig. 1 Detection and demarcation of preablation margins with RCM imaging. (a) Example of an $8 \mathrm{~mm} \times$ $8 \mathrm{~mm}$ mosaic captured at the dermal-epidermal junction. The red dashed lines from 12 to 6 and 3 to 9 o'clock indicate the quadrants utilized to keep localized record of the tumor location and estimated depth. The quadrants are defined in clockwise orientation. The tumor margins are delineated with the dashed yellow boundary, and the corresponding selected regions for control and ablation are shown (this is only for those 10 cases in which a thin excision was performed immediately postablation for histology correlation). (b) Images at different depths from a stack, captured at the location of the red square in (a), contain features of BCC used for estimation of tumor depth: at $\sim 60 \mu \mathrm{m}$ (epidermal layer) enlarged blood vessels (yellow arrows); at $100 \mu \mathrm{m}$ enlarged blood vessels (yellow arrows) and tumor islands (red arrows); and at $\sim 160 \mu \mathrm{m}$ enlarged blood vessels (yellow arrow), tumor island (red arrow), and cell palisading (green arrow) surrounding the tumor island. 
directly on the patient before, during, and after the ablation treatment.

\subsubsection{Lateral and deep tumor margins imaging preablation}

Figure 1 shows the protocol. The preablation imaging was guided by the surgeon's initial visual demarcation of the clinical margins of the lesion using surgical pen ink. Three RCM mosaics $(8 \mathrm{~mm} \times 8 \mathrm{~mm})$ centered within the lesion were collected with a Vivascope 1500 , one above the basal layer in the epidermis, one including the basal layer at the dermalepidermal junction, and one below in the underlying papillary dermis, to estimate the general location, lateral margins, and depth of tumor. The more exact location of the tumor margins was subsequently recorded by further demarcating the lesion area (i.e., to-be-imaged area) in four quadrants, using 12, 6, 9, and 3 o'clock as reference points denoted by surgical marks. To facilitate RCM navigation, we placed adhesive paper rings outside the wound margins (paper fibers are visible with high contrast in RCM). ${ }^{25}$ Stacks of images, with $5-\mu \mathrm{m}$ axial separation, were collected in each quadrant where tumor was detected to more exactly estimate the corresponding depth. Half of the lesion was selected for ablation and the remaining intact half served as a control. The two halves were separated by a line inkmarked on the skin of the patient by the surgeon (Fig. 1). Each quadrant of the imaged area was ablated with a number of passes to achieve a depth of ablation (using our Table 1) corresponding to the estimated depth of tumor by RCM. When the lesion was larger than $8 \mathrm{~mm} \times 8 \mathrm{~mm}$, the surrounding tumor served as a control as well.

In Fig. 1(a), an example of a mosaic collected at the epidermal layer (at $\sim 60-\mu \mathrm{m}$ depth from the top layer), in which presence of BCC tumors (dark silhouette delineated by the dashed yellow line) was detected at the center of the mosaic. In Fig. 1(b), three images from a stack of 30 images collected at the location of the red square in Fig. 1(a) at $\sim 60,100$, and $\sim 160 \mu \mathrm{m}$ show features of BCCs, such as enlarged blood vessels (yellow arrows), tumor islands (red arrows), and palisading (green arrow) around the tumor island. A detailed description of the characteristic features of BCCs under RCM imaging in intact tissue can be found in our previous work. ${ }^{23}$

\subsubsection{Selection of number of passes and depth of ablation}

The estimated depth of tumor provided by the RCM stacks was used to select the number of passes. This was based on our previous study results in human skin specimens ${ }^{19,20}$ and the initial testing on patients. ${ }^{17}$

\subsubsection{Topical application of contrast agent postablation}

Figure 2(a) show the geometry of the wound. Postablation, the surface of the shallow wound was swabbed with $35 \%$ aluminum chloride solution for $30 \mathrm{~s}$ using sterile applicators, followed by filling the cavity with sterile gel. More details of the technique have been reported in our earlier papers. ${ }^{17}$ Aluminum chloride was previously discovered to enhance nuclear contrast in RCM images $^{21}$ and its development for the intraoperative detection of residual BCCs in surgical wounds has been reported in previous studies. $^{21,26,27}$ Topical application for $30 \mathrm{~s}$, with concentration of $35 \%$, was found to be optimal. ${ }^{26,27}$ Higher concentrations and/or longer application produces dehydration and necrosis in tissue, whereas lower concentrations and/or shorter times does not brighten nuclear morphology.

\subsubsection{Lateral and deep margins imaging postablation}

Figure 2 shows the protocol for imaging in the wound postablation. Imaging was at 8 frames/s while using a Vivascope 3000 to rapidly assess the area of ablation. Videos were acquired along the superficial epidermal and deep dermal margins starting at the 12 o'clock position. The 12 o'clock position was surgically marked on the patient's intact skin by the surgeon preablation. The wound was divided into quadrants, e.g., 12 to 3,3 to 6,6 to 9 , and 9 to 12 to keep records of the exact location of the imaging findings. Stacks of images were captured at those suspicious areas where the potential presence of residual tumor was detected. The stack allows visualization of the suspicious features as a function of depth. After the stacks were captured, the microscope was switched back to video mode to continue imaging and capturing videos of the remaining margins.

\subsubsection{Video mosaics}

The captured videos were processed (i.e., sequence of video images stitched together) to generate video mosaics to display

Table 1 Depth of ablation versus number of passes for fluence of $25 \mathrm{~J} / \mathrm{cm}^{2}$. In this study, fluence was constant at $25 \mathrm{~J} / \mathrm{cm}^{2}$. The small differences between the ex vivo and in vivo measurements are explained in the text (Sec. 3.2).

No. of passes $25 \mathrm{~J} / \mathrm{cm}^{2}$

\begin{tabular}{|c|c|c|c|}
\hline \multirow[b]{2}{*}{ Depth of ablation } & \\
\hline & 4 to 5 passes & 6 to 7 passes & 8 to 11 passes \\
\hline Measured in vivo with histology & $\begin{array}{l}\sim 80 \text { to } 122 \mu \mathrm{m} \text { complete } \\
\text { epidermis }\end{array}$ & $\sim 130$ to $160 \mu \mathrm{m}$ papillary dermis & $\begin{array}{l}\sim 170 \text { to } 200 \mu \mathrm{m} \text { partial reticular } \\
\text { dermis }\end{array}$ \\
\hline Number of lesions & 2 & 3 & 5 \\
\hline Estimated in vivo with $\mathrm{RCM}$ & $\begin{array}{l}\sim 90 \text { to } 136 \mu \mathrm{m} \text { complete } \\
\text { epidermis }\end{array}$ & $\begin{array}{l}\sim 130 \text { to } 160 \mu \mathrm{m} \text { papillary dermis } \\
\text { and partial reticular dermis }\end{array}$ & $\begin{array}{l}\sim 180 \text { to } 200 \mu \mathrm{m} \text { partial deeper } \\
\text { reticular dermis }\end{array}$ \\
\hline Number of lesions & 9 & 15 & 10 \\
\hline Measured ex vivo & $\begin{array}{l}\sim 85 \text { to } 120 \mu \mathrm{m} \text { papillary dermis to } \\
\text { partial deeper reticular dermis }\end{array}$ & $\begin{array}{l}\sim 150 \text { to } 170 \mu \mathrm{m} \text { partial reticular } \\
\text { dermis }\end{array}$ & $\begin{array}{l}\sim 170 \text { to } 220 \mu \mathrm{m} \text { partial deeper } \\
\text { reticular dermis }\end{array}$ \\
\hline
\end{tabular}



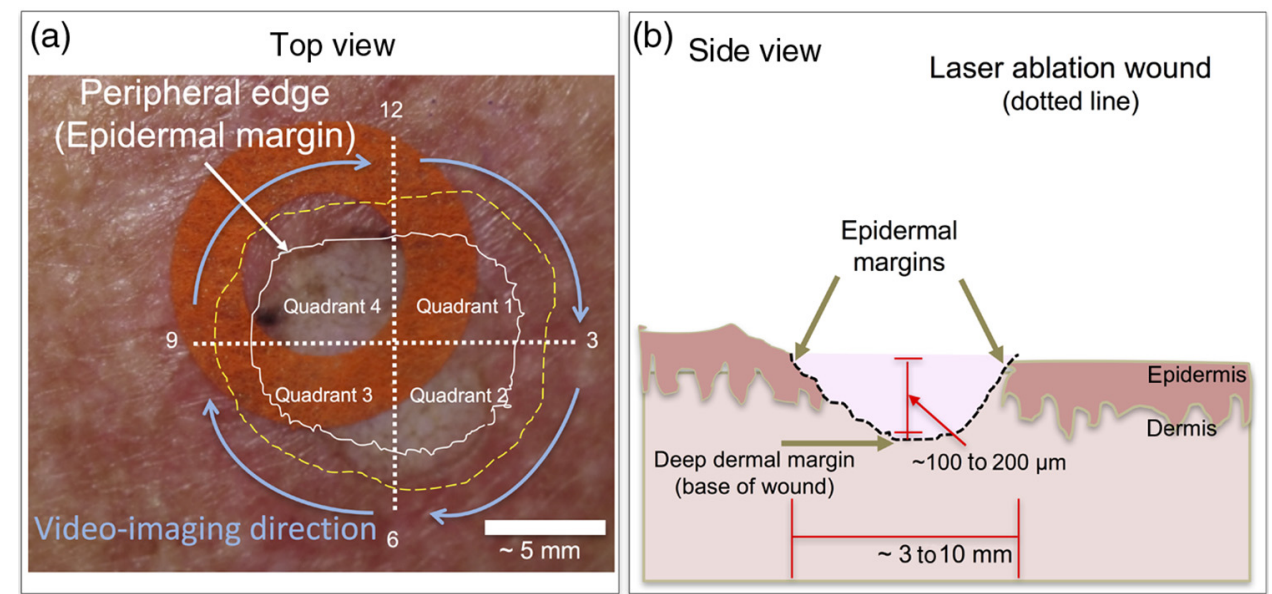

Fig. 2 Postlaser-ablated wound topography and RCM video-imaging approach. (a) The lines and arrows on the clinical photograph illustrate the approach of using a paper ring (orange ring) to isolate each quadrant of the wound for imaging and provide a more precise location of findings with respect to the clinical coordinates established by the surgeon. The wound edge (white boundary) is used as a reference for imaging along the epidermal margins, and imaging of the peripheral dermal margins below the edge (yellow dotted boundary) and at the center of the wound. The blue arrows illustrate that videos were acquired along the epidermal margin, starting from the 12 to 3 , and proceeding from 3 to 6,6 to 9 , and 9 to 12 o'clock positions. (b) A cartoon of the side view of a postlaser-ablated wound showing the epidermal margins and deep dermal margins (base of wound).

a field of view 1-mm wide with the length defined by the length of the video that covered the entire epidermal margin. For small wounds ( 3 to $5 \mathrm{~mm}$ in diameter), videos were acquired in individual quadrants starting at the 12 o'clock position in a clockwise direction [Fig. 2(b)]. A single video was collected of the peripheral epidermal margin of each quadrant (from 12 to 3,3 to 6, 6 to 9 , and 9 to 12 o'clock), displaying intact surrounding skin and exposed epidermis. The corresponding video mosaics for each quadrant have dimensions of $\sim 1 \mathrm{~mm} \times 7.5 \mathrm{~mm}$. For small wounds, the imaging of the periphery was followed by a video captured at the central portion of the wound (base of wound). For larger wounds ( $>5 \mathrm{~mm}$ in diameter), additional videos of the deep dermal margins were collected in the center of each quadrant. The videos were collected in clockwise circular paths. Some of the videos contained spatial overlap due to the manually controlled video capture approach that led to variable lengths of the video mosaics. More details of the software and implementation can be found in other papers. ${ }^{24,26}$

Confirmation of clearance of BCCs on the second set of 34 lesions was based on real-time examination of the videos as they were captured on patients. At present, processing videos and creating video mosaics requires $\sim 3$ to 5 min per quadrant (for a length of $\sim 1 \mathrm{~mm} \times \sim 7.5 \mathrm{~mm}$ ). Since the length of the videos for deep dermal margins was variable due to the uncontrolled manual video capture approach, the processing time for video mosaics was 2 to $5 \mathrm{~min}$. As such, video mosaics were not available in real time during imaging on patients and could not be used to determine clearance of margins during the laser ablation treatment. However, they were employed later on for further image analysis, to correlate imaging findings to histopathology results for the first 10 lesions, and to further confirm the original real-time (i.e., immediately postablation) findings on the remaining 34 lesions.

Figure 3 shows the comprehensive perioperative RCM imaging-guided laser ablation procedure, which mimics the procedure for frozen pathology-guided Mohs surgery. Preablation: (a)

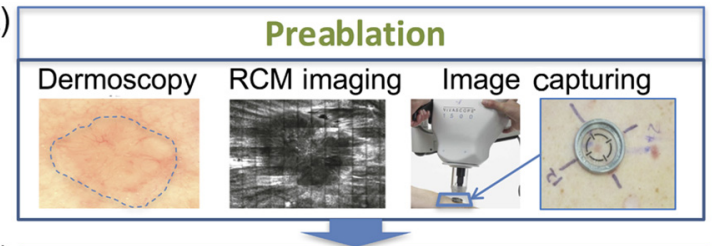

(b)

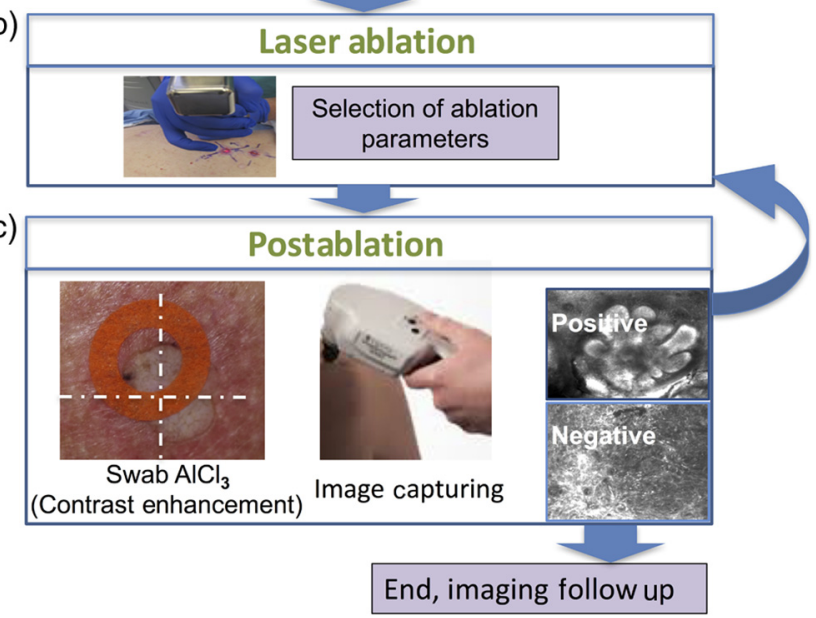

Fig. 3 Laser ablation guided with perioperative feedback from RCM imaging of BCC tumor morphology with cellular-level resolution. (a) Preablation: mosaics and stacks are acquired to estimate BCC margins and tumor depth preablation. The use of Vivascope 1500 with a tissue contact ring to acquire mosaics and stacks preablation (top row, two panels on the right). (b) Laser ablation: number of passes is selected based on the estimated depth of tumor to obtain the sufficient depth of ablation for complete removal of tumor. (c) Postablation: videos are acquired with a Vivascope 3000 to verify clearance of margins and stacks to review suspicious areas as a function of depth after ablation (bottom). Video mosaics are generated to visualize larger fields of view postablation. The ablation guided by $\mathrm{RCM}$ imaging protocol mimics the standard procedure of Mohs surgery guided by frozen pathology. 
RCM mosaics and stacks are acquired to estimate BCC margins and tumor depth preablation; laser ablation: number of passes are selected based on the estimated depth of tumor to obtain the sufficient depth of ablation for complete removal of tumor; and postablation: videos are acquired with a 3000 Vivascope (handheld RCM) to verify clearance of margins and stacks to review suspicious areas as a function of depth after ablation (bottom). Video mosaics are generated to visualize larger fields of view postablation.

\subsection{Imaging-Guided Laser Ablation with Immediate Histology Confirmation}

Ten BCCs lesions were treated and the imaging results immediately confirmed with histology. An $8 \mathrm{~mm} \times 8 \mathrm{~mm}$ area, centered within the lesion containing tumor, was selected for RCM imaging and ablation. The tumor in the surrounding area served as control when the lesion was greater in size. If the lesion was smaller than $8 \mathrm{~mm} \times 8 \mathrm{~mm}$, half of the lesion was selected for ablation with the remaining portion serving as control. RCM imaging-guided laser ablation protocol was executed and the postablation imaging detected the presence of residual tumor or complete clearance. Immediately afterward, our Mohs surgeon (coauthor CSJC) performed a thin excision and sent it to the laboratory for histology preparation to validate the imaging findings.

\subsubsection{Correlation to and validation against histopathology}

The excised tissue was embedded for preparation of hematoxylin and eosin (H\&E)-stained sections. Vertical sections (oriented perpendicular to the tissue surface) were prepared to visualize the location of the residual tumor, if any (or, clearance, if none). The excised tissue was oriented so that the frozen sections would include both ablated and control regions. The presence and appearance of residual BCCs (or clearance) in H\&E sections was analyzed under light microscopy and compared with postablation RCM findings.

\subsubsection{Depth of ablation}

Lesions treated with 4 to 5,6 to 7 , and 8 to 9 passes were chosen for performing depth of ablation measurements. A frozen section containing the center of the ablated area was selected. Two measurements were made from each section: one at the shallowest location (edge of postablated wound) and another at the deepest (center) of the ablated region relative to the adjacent nonablated skin, to provide a range for the depth of ablation values.

\subsection{Imaging-Guided Laser Ablation with Immediate Reflectance Confocal Microscopy Confirmation and Longer-Term Follow-Up (No Histopathology)}

Thirty four superficial and early nodular BCCs were treated with RCM imaging-guided laser ablation for complete tumor removal. All lesions were diagnosed clinically and dermoscopically, and also confirmed with BCC features under RCM before the ablation. The number of passes was guided by the imaging and imaging-based measurement of depth until clearance of tumor was confirmed with immediate imaging. No histology was performed. This method was limited to relatively small lesions of diameter $<10 \mathrm{~mm}$.
Follow-up imaging is being currently conducted to measure the accuracy of the imaging-guided approach to detect clearance of tumor postablation, and to monitor the longer-term efficacy of the ablation treatment and achieve follow-up periods of at least 12 months for all treated lesions. Video imaging and mosaicking of the peripheral epidermal and dermal margins and the deeper dermal margins at the center region (base of wound) of the treated lesions is being conducted at 1 to 3,6 , and 18 months after treatment.

\subsection{Assessment Depth of Ablation, Uptake of Contrast Agent, Reflectance Confocal Microscopy Images, Video-Mosaics}

Image stacks were collected (where presence of tumor was detected) to estimate tumor depth, which then allowed us to select the appropriate number of passes to provide the depth of ablation required for complete removal of the tumor.

The qualitative evaluation of postablation imaging was focused on the uptake of contrast agent and detectability of tumor and normal features as a function of depth of ablation and number of passes. Three clinicians (coauthors CSJC, MC, and $\mathrm{OY}$ ) with experience in reading and analyzing confocal images of BCCs evaluated the quality of RCM images, stacks, videos, and video mosaics. The overall quality was qualitatively assessed as acceptable or not for resolution, contrast, and visualization of nuclear and morphological detail. The visualization of bright nuclear morphology in the exposed peripheral epidermal margin, dermal structures, such as collagen bundles, hair follicles, eccrine glands, and inflammatory cells in the deeper dermal margin (base of wound), as well as the presence or absence of tumor in each quadrant of the wound were analyzed.

\section{Results}

We determined that, overall, RCM imaging, with the topical use of aluminum chloride in Er:YAG laser-ablated wounds, is feasible. Nuclear labeling in epidermal margins was consistent and repeatable in all the imaged wounds, enhancing nuclear brightness that facilitated the detection of presence or absence of residual tumor after ablation.

The acquisition of videos with the handheld microscope (Vivascope 3000) is reasonably rapid, reasonably efficient, and allows imaging of the superficial epidermal and deep dermal margins of the shallow wound cavity. The acquisition of videos required $\sim 5 \mathrm{~min}$ for wounds of $8 \mathrm{~mm}$ in diameter. Conversion of videos to video mosaics requires a bit more time; $\sim 10$ to 15 min a video that covers the complete peripheral margin. The video mosaics allow visualization of the surrounding tissue morphology and cellular structures, and detection of the presence or absence of tumor. What we observed in the video mosaics provided confirmation of the real-time observations in the videos when they were being captured in the clinic during treatment.

\subsection{Uptake of Contrast Agent in the Superficial Epidermal and Deep Dermal Margins of the Ablated Wounds}

In Fig. 4, a video mosaic generated from a video captured from the surface of a laser ablation wound is shown.

Figure 4(a) shows an example video-mosaic postablation, in this case with seven passes of $25 \mathrm{~J} / \mathrm{cm}^{2}$. In Fig. 4(b), details of the superficial margins are shown. From left to right, we see 

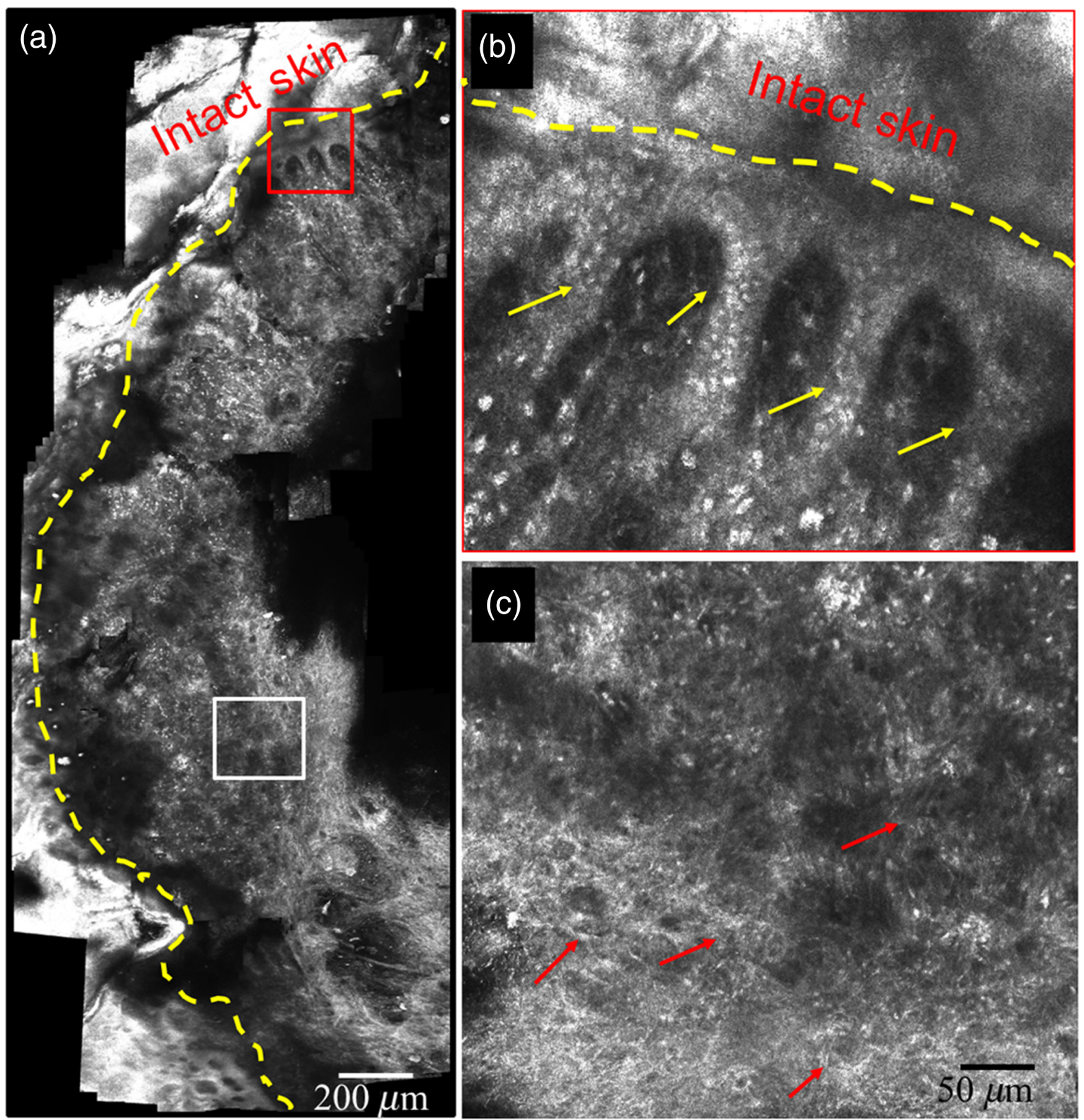

Fig. 4 Video mosaic of a postablation wound that was produced from an RCM imaging video. (a) Clearance of tumor is seen after the removal of $\sim 130 \mu \mathrm{m}$ of tissue with six passes of $25 \mathrm{~J} / \mathrm{cm}^{2}$. (b) enlarged view [of the red square in (a)] of the peripheral epidermal margin showing intact skin (left of yellow dotted line), bright nuclei of the basal cells, due to uptake of contrast agent (aluminum chloride), at the dermal-epidermal layer (shown with the yellow arrows); and (c) enlarge view of collagen fibers in the normal dermis at the deeper margin (inside of the white square, indicated by red arrows).

intact skin, bright nuclei of basal cells at the epidermal margin, suggesting the uptake of contrast agent (yellow arrows) and in Fig. 4(c), collagen fibers in normal dermis in the deeper dermal margin (red arrows).

Overall, aluminum chloride produced repeatable and consistent brightening of nuclear morphology and enhanced the contrast and detectability of residual BCC and normal structures after laser ablation with four to nine passes of $25 \mathrm{~J} / \mathrm{cm}^{2}$ fluence.
However, improper (such as excess or no uniform) application or tissue heterogeneity was found to sometimes produce saturation artifacts. In three postablation imaging cases, we observed saturation due to the contrast agent, preventing the distinction of normal from abnormal cellular patterns. However, as seen in Fig. 5, the nuclei, cellular detail, and dermal morphology, such as inflammatory cells, hair follicles, collagen fibers, eccrine, and sebaceous glands, could be differentiated. 

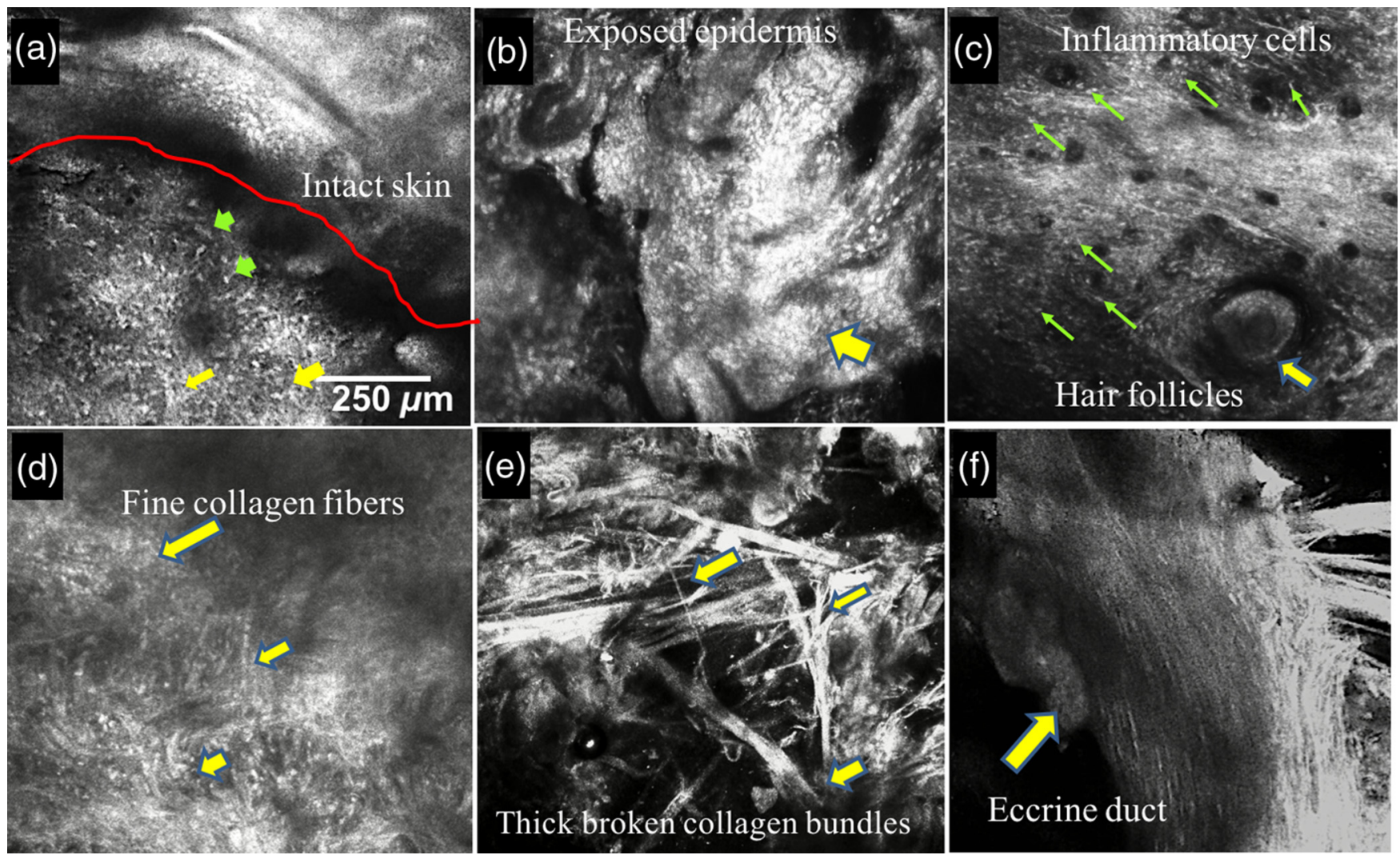

Fig. 5 RCM images showing morphologic structures that are typically seen in postablation wounds: (a) the wound edge (red line), with intact skin (top) showing the honeycomb pattern of dark nuclei and bright cytoplasm of the epidermal cells, at the wound edge bright nuclei (green arrows) of the exposed epidermis (enhanced by the aluminum chloride) and deeper in the dermis (lower side) collagen fibers at the dermis level (yellow arrows); (b) exposed of epidermis in the wound with cobblestone pattern of bright nuclei (yellow arrow); (c) inflammatory cells (green arrows), bright rounded small nuclei as seen in the deep dermal margin and hair follicles (lower yellow arrow); (d) bright fibrillar structures corresponding to fine collagen bundles; (e) thick collagen bundles seen in the deep dermis; and (f) eccrine glands (yellow arrow). Scale bar is $250 \mu \mathrm{m}$ for all images.

Thirty one out of thirty four laser-ablated wounds could be visually graded as being of clinically acceptable imaging quality, in terms of resolution, contrast, and detectability of features. Those graded as being of unacceptable image and/or video quality were due to artifacts, such as bubbles (created by an excess amount of gel and/or smearing of the gel during imaging), which resulted in completely or partially blocking the field of view, and saturation of brightness and loss of contrast, which compromised the distinction of cellular structures. Other reasons for low-quality videos included loss of information (dark images) due to sudden changes in wound topography, variability in operator movement and speed causing motion blur, and variability of pressure against skin, all of which produced discontinuities in the videos during the imaging.

\subsubsection{Epidermal margin: at the peripheral edge (at the rim) of the ablated wound}

At the periphery of the wound, the direct exposure to topically applied aluminum chloride produced brightening of nuclei. In the intact skin surrounding the wound, nuclei appear dark, as is normally seen during imaging in vivo. (The intact stratum corneum prevents diffusion of aluminum chloride into the epidermis.) The difference between bright nuclear details in the exposed epidermis against the dark nuclear details in intact skin was easily distinguished and facilitated the detection of the edges of each wound during imaging. Figure 4(b) shows an example of the transition from intact to exposed epidermis (across the yellow dotted line from left to right).

\subsubsection{Peripheral dermal margin: the superficial papillary dermis (below the epidermal rim) of the ablated wound}

The dermal-epidermal junction appears as semicircle patterns of bright cells, contrasting with the darker background [yellow arrows in Fig. 4(b)]. At deeper levels in the wound, collagen bundles are also visualized [red arrows in Fig. 4(c)].

\subsubsection{Deep dermal margin: reticular dermis at the base of the ablated wound}

In the deeper layers, the base of the wound appeared more uniform with less cellular structures but containing bright fibrillary structures that corresponded to collagen fibers.

\subsection{Depth of Ablation}

The depth of ablation values range from 80 to $122 \mu \mathrm{m}$ for 4 to 5 passes, 140 to $170 \mu \mathrm{m}$ for 6 to 7 passes and 170 to $200 \mu \mathrm{m}$ for 8 to 9 passes as a function of passes. In Table 1, these values are shown as a function of the number of passes. The depth of 
Table 2 Imaging guided laser ablation with correlation to histology in $10 \mathrm{BCC}$ lesions on six patients.

\begin{tabular}{llcccc} 
Lesion & \multicolumn{1}{c}{ BCC subtype } & $\begin{array}{c}\text { Estimate } \\
d \text { depth }\end{array}$ & $\begin{array}{c}\text { Number } \\
\text { of passes }\end{array}$ & $\begin{array}{c}\text { Residual BCC in } \\
\text { assessed by RCM }\end{array}$ & $\begin{array}{c}\text { Residual BCC on } \\
\text { vertical histology } \\
\text { postablation }\end{array}$ \\
\hline 1 & Superficial and early nodular & 175 & 9 & Yes & Yes \\
2 & Superficial and early nodular & 180 & 8 & Yes & Yes \\
3 & Superficial intradermal component & 210 & 9 & Yes & Yes \\
4 & Superficial and infiltrative nodular & 180 & 9 & Suspicious & Yes \\
5 & Superficial and early nodular & 150 & 8 & No & No \\
6 & Superficial & 85 & 5 & No & No \\
7 & Superficial & 88 & 5 & No & No \\
8 & Superficial & 116 & 6 & No & No \\
9 & Superficial & 125 & 7 & No & No \\
10 & Superficial and early nodular & 135 & 7 & No & No \\
\hline
\end{tabular}
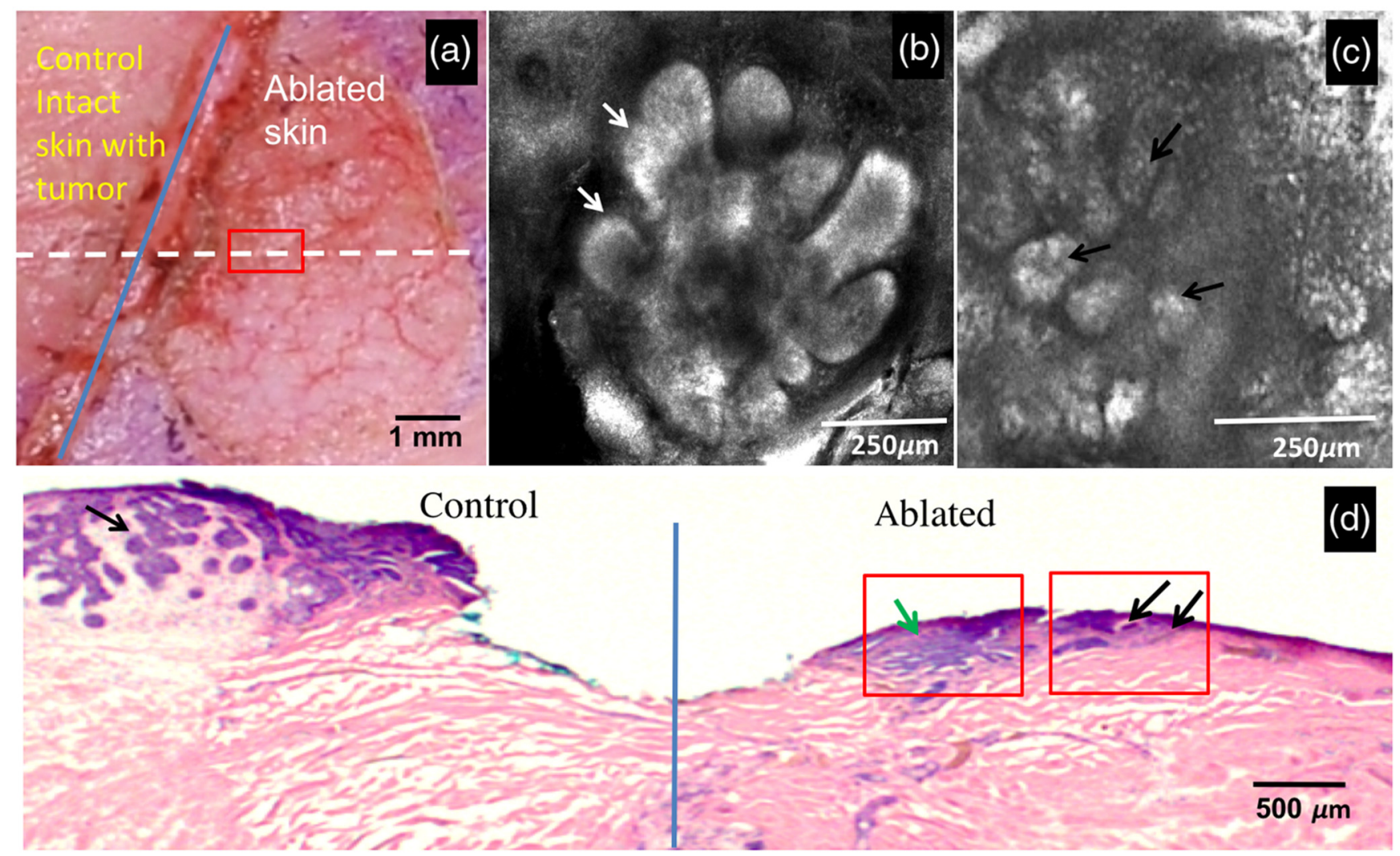

Fig. 6 In vivo detection of clearance of tumor or presence of residual tumor postablation, for ablation with four passes at fluence of $25 \mathrm{~J} / \mathrm{cm}^{2}$. (a) A clinical photograph image of the lesion postablation, with a solid blue demarcation line, showing the intact area of the lesion that contains tumor (left, nonablated control area) and the ablated area (right); (b)-(c) RCM image of residual nodular BCC (white and black arrows) at the peripheral epidermal margin, located inside the solid red square in (a); (d) H\&E-stained histopathology that confirms RCM findings, showing intact tumor [black arrow at the left of the solid blue line, corresponding to the nonablated control area in (a)] and the surgical margin (made by Mohs surgeon JC and labeled with light blue ink before treatment); and residual tumor in the ablated area (right of the solid blue line inside the solid red squares) corresponds to that in (b) (white arrows) and (c) black arrows. The vertically sectioned histology allows visualization of the residual tumor (green and black arrows) at the edge of the crater-shaped wound validating the continuity of the tumor from the nonablated control area to the ablated region. 
ablation measurements from our previous ex vivo study is also shown. In general, there is reasonable agreement between the measurements in vivo and those ex vivo. Variations between in vivo and ex vivo measurements are attributed to variations in skin sites and hydration conditions on patients, the presence of stratum corneum, and motion artifact due to the laser beam being slightly noncollimated and slightly diverging (the ablation was performed manually, such that slight variations in the distance between the laser scanner and the patient could result in slight variations in fluence). Nonetheless, the results indicate that the depth of ablation versus number of passes measurements ex vivo can be a reasonable initial "look up table" guide for choosing ablation parameters (fluence, number of passes) based on RCM-guided measurements of depth of tumor in vivo.

\subsection{Correlation to Histology in the First Six Patients (10 Basal Cell Carcinomas) for Presence or Absence of Tumor}

Table 2 shows the selected laser ablation parameters (number of passes for fluence of $25 \mathrm{~J} / \mathrm{cm}^{2}$ ) based on depth of tumor that was estimated by preablation RCM imaging. For all lesions, a thin excision was taken for histology postablation. In 3 out of 10 lesions, residual BCCs were detected by RCM postablation. For those cases, the residual tumors were not further removed with laser ablation, but were surgically excised, and then confirmed and correlated to histology. The correlation of imaging findings to histology resulted in three true positives, six true negatives, and one false positive. For the false positive case, the presence of saturation artifacts in the imaging
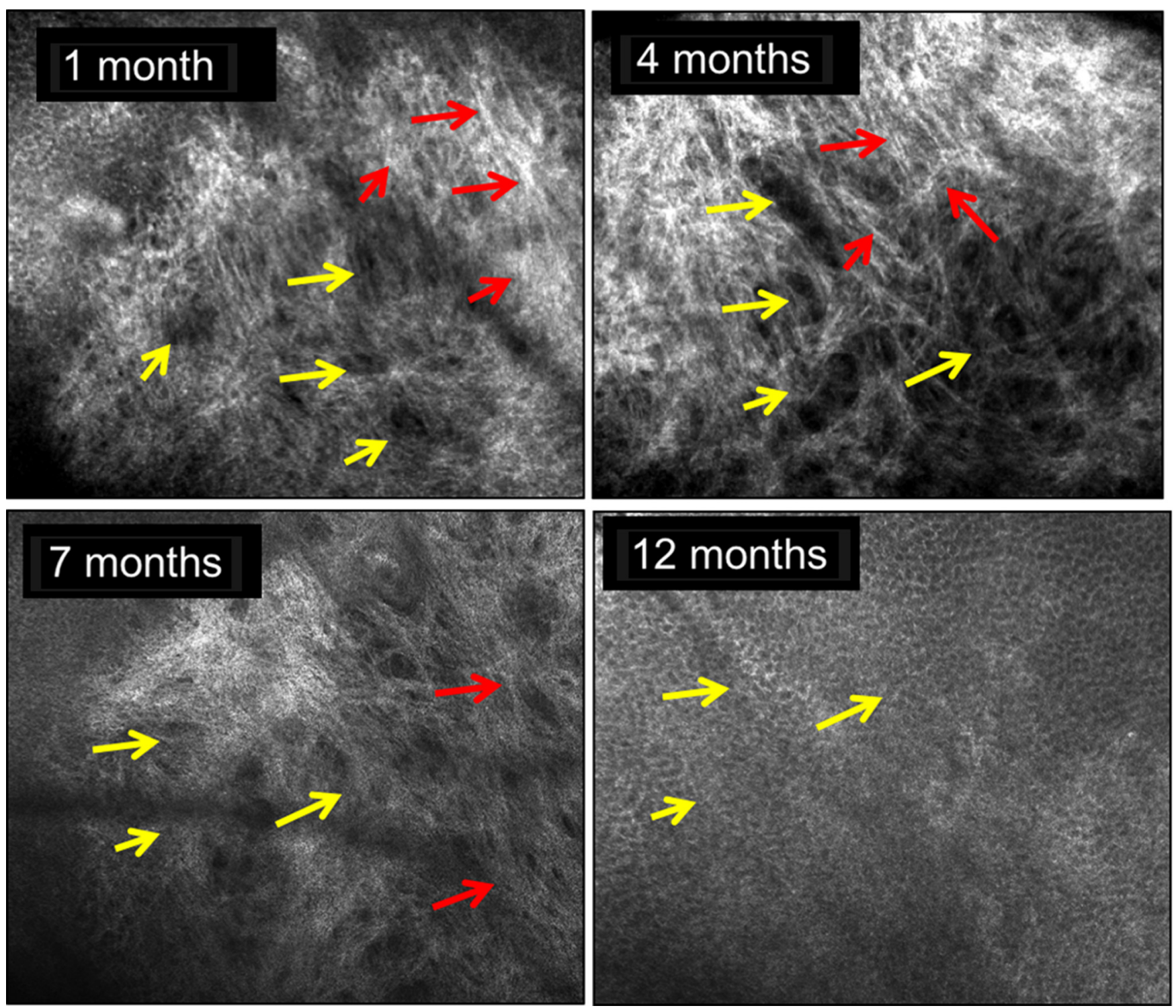

Fig. 7 Follow-up RCM imaging of lesion number 3 (superficial BCC) at 1, 4, 7, and 12 months after ablation. In this case, ablation was with six passes at fluence of $25 \mathrm{~J} / \mathrm{cm}^{2}$. Presence of enlarged blood vessels (yellow arrows) and amorphous collagen fibers (red arrows) at 1 and 4 months (top) were observed as part of the wound healing process. At 7 months, presence of enlarged blood vessel (yellow arrows) was still observed with thickened collagen fibers (red arrows). Normal skin patterns without features of BCC were seen at 12 months of postablation. Dark nuclei patterns (yellow arrows, lower right) similar to that observed in normal skin epidermis. 
prevented our clinical imaging reading expert (coauthor MC) from reaching conclusive identification of the residual tumor.

An example of detection of residual tumor postablation with four passes of $25 \mathrm{~J} / \mathrm{cm}^{2}$ is shown in Fig. 6. In Fig. 6(a), a clinical photograph (routinely taken in the clinic) of the lesion is shown, along with a solid blue line on the surgical scoring (performed by the Mohs surgeon) demarcating the ablated region (right side) and intact region as control (left). Postablation imaging detected residual tumor at the peripheral margins. Figures 6(b)-6(c) show RCM images of typical features of $\mathrm{BCC}$ indicating the presence of residual tumor, corresponding to the region enclosed in the solid red rectangle in Fig. 6(a). In (b), the high density nuclei enhanced in contrast by the aluminum chloride form a flower shaped structure that is typical of BCCs surrounded by a palisading pattern (white arrows). Figure 6(c) shows small island of tumor formed by bright nuclei (black arrows). In Fig. 6(d), an H\&E section taken at the location of the white dashed line in Fig. 6(a) is shown. At the left side, the intact tumor (black arrow) intentionally left for control and the surgical margin (the crater with the edge labeled with light blue ink) serves as a reference to identify the ablated region. At the right side, the green and black arrows indicate details of the presence of residual BCCs at the epidermal margins confirming the findings shown in Figs. 6(b) and 6(c), respectively.

\subsection{Image-Guided Laser Ablation with Imaging Follow-Up on Remaining 15 Patients (34 Basal Cell Carcinomas)}

Initial follow-up results are available for 26 superficial, six superficial and early nodular, and two early nodular BCCs that were treated with only the RCM imaging-guided laser ablation approach (i.e., no histopathology). The number of passes varied from 5 to 11 as shown in Table 1 (row 2). After the first ablation treatment RCM imaging detected clearance of tumor in 27 lesions and residual tumor in seven lesions (two treated with eight passes and five treated with 5 to 6 passes) for which a second treatment (of 2 to 4 passes) was applied to attain clearance of tumor. The results for 31 lesions show that the uptake of $\mathrm{AlCl}_{3}$ as contrast agent to label nuclei appears to be effective and consistent in wounds, with estimated depths of ablation from 80 to $200 \mu \mathrm{m}$. However, in three cases (ablated with 6 to 8 passes), saturation was observed in the images that challenged the detection of nuclear structures. All lesions are currently being followed up and the ablation treatment monitored with imaging at 1 to 3 (7 lesions), 6 to 7 (12 lesions), and 10 to 21 (15 lesions) months. The monthly time points tend to vary a bit due to patients' schedule for the follow-up appointments.

To date, 31 lesions have shown good cosmetic outcome and clearance of tumor under RCM images. Only two of the lesions (superficial and superficial/nodular types of BCCs at 7 and 17 months of follow-up, respectively) have shown recurrence after 6 months, respectively, where imaging showed features indicating presence of BCC. In one lesion (superficial type of $\mathrm{BCC}$ at 7 months follow-up), persisting large blood vessels and elongated nuclei patterns at the epidermis layer were observed as suspicious features of presence of BCC.

At early stages, most of the lesions were more raised and slightly erythematous. After 12 months, most of the followed lesions were the same color as surrounding skin or pink. Hypopigmented macules in five lesions ( $>16$ months of follow-up), pink macules in two lesions ( $>16$ months of follow-up), pink papules (raised) in three lesions ( $>6$ months of follow-up), erythematous macules in three lesions ( $>6$ months of follow-up), and hypertrophic scarring in one lesion (at 2 months of follow-up) were observed.

An example of follow-up imaging, collected at 1, 4, 7, and 12 months, is shown in Fig. 7, for one of the lesions (with 20 months of follow-up) representing the evolution of treated superficial type of BCC. The regrowth skin layers are seen at the first follow-up. However, significant presence of enlarged blood vessels (yellow arrows) and amorphous and thin collagen fibers (yellow arrows) are seen at 1 and 4 months. At 7 months, more normal dermis is visualized still presence of enlarged blood vessels with thickened collagen fibers (red arrows) were observed (Fig. 7-left bottom). The appearance and morphology of the epidermal layers were seen normal compared to untreated surrounding normal skin at 12 months (Fig. 7-right bottom).

\section{Discussion}

This paper reports another step in our ongoing development of RCM imaging methodologies to guide treatment of BCCs on patients. In this case, the treatment is ablation with an Er: YAG laser. Ablation vaporizes tissue such that none is available for traditional pathology, and the treatment is guided purely with imaging. This study represents our initial clinical experience using RCM imaging in superficial ablated wounds. The imaging addresses, to some extent, the current major limitation of lack of histological confirmation as reported in previous studies. As described herein, RCM imaging can eloquently map lateral and deep tumor margins directly on patients preablation, helping refine the laser ablation parameters (optimal fluence and/or number of passes) for precise destruction of BCCs without excessive loss of surrounding normal tissue, as well as detect residual tumor or confirm clearance postablation, again, directly on patients.

The initial pilot study on 10 lesions with histology confirmation allowed us to explore the efficacy of ablating superficial and early nodular BCCs when guided by RCM in vivo. The selection of a control region containing $\mathrm{BCC}$ within the lesion demarcated the intact (nonablated) tumor versus ablated BCCs helped for correlation and validation of the RCM imaging findings to histology. Out of 10 lesions, only one case was misread, as a false positive, due to saturation artifacts. Admittedly, 10 cases can be considered a small number, but, nonetheless, these first results on patients confirm the findings from our earlier ex vivo studies and build upon our initial experience on patients.

Three residual BCC cases (lesion numbers 1 to 3 in Table 2) detected with postablation RCM were confirmed with histology. Those cases had an early nodular component. In these cases, we observed the presence of bright tumor islands (enhanced due to the use of aluminum chloride), elongated nuclei, and clefting in RCM images. The other case (lesion number 4 in Table 2) that showed suspicious tumor on RCM was a BCC with an infiltrative component. Infiltrative BCCs are often deeper than RCM can image and this component was not detected before ablation. (Infiltrative, micronodular, and mixed types of BCCs were not included in this study.) After ablation, too, residual infiltrative BCCs are often difficult to detect due to their small size and the limited specificity of reflectance contrast in RCM images. Furthermore, it is important to note that, after several passes, ablated epidermal cells under RCM can sometimes appear elongated, similar to the polarized nuclear morphology in BCCs and 
akin to that seen on histology. Therefore, it is ideal to balance the proper amount of ablation and thermal coagulation by controlling the number of passes and fluence to effectively treat tumor while preserving enough tissue architecture to be able to image and diagnose under RCM. This finding could also explain the challenge in identifying residual BCC in the fourth case, where the features of tumor were not strikingly evident in RCM images but were clearly identified in histology. However, since these were among the first-treated lesions with the RCM imaging approach, the initial lack of experience in reading images in ablated tissue may have also played a role in the missed detection of BCCs in postablated tissue.

The subsequent study on 34 lesions, with only RCM imaging but no histopathology, allowed us to further explore and test this approach on a larger number of patients. RCM imaging found residual $\mathrm{BCC}$ for seven of the 34 treated lesions after ablation. For these lesions, an additional second set of 2 to 4 passes was required to achieve clearance of $\mathrm{BCC}$. The failure to complete removal of tumor after the first set of passes may have been due to the undercalculation of the depth of tumor by RCM. This may be a consequence of the limited depth of RCM imaging and the decrease in resolution in the deeper layers of the skin $(\sim 150$ to $200 \mu \mathrm{m}$ ), which prevents accurate and repeatable estimation of the deeper margins and depth of tumors. Clearance in 31 lesions over a follow-up range of 1 to 21 months (mean follow-up 13 months) has been observed. However, for the seven lesions with 1 to 3 months follow-up, the results are still premature to clinically state cure of tumor at such short follow-up times. At such short times, features innate to the healing and scarring process (higher presence of blood vessels, inflammatory cells, etc.) appear under the imaging and may result in a biased evaluation. Nonetheless, this limitation notwithstanding, we have, to date, seen promising outcomes and clinicians continue the follow-up imaging to complete the follow-up to more than 12 months for all 34 lesions for complete clinical evaluation.

To overcome RCM's limited depth of imaging, a multimodal approach may be considered. Optical coherence tomography (OCT) and ultrasound imaging have been proven to detect BCCs at depths of $\sim 1 \mathrm{~mm} .{ }^{27,28}$ Combining RCM imaging with OCT or ultrasound will likely address this limitation. The optical synergy of OCT and RCM, in particular, facilitates the integration of the two modalities into a single device. Indeed, preliminary benchtop instrumentation work and recent development of a handheld prototype has shown the possibility of OCT to provide an integrated complementary tool to RCM for the purpose of imaging deeper margins and to improve estimation of tumor depth preablation. ${ }^{29,30}$

Another limitation of this first study is the mostly uncontrolled manual operation of the RCM device and inability of the imaging operator to manually cover the entire deep dermal margins (surface of the wound base). These limitations may be solved with the development of a smaller RCM device, with a smaller objective lens, and a less manual and more automated approach to ensure complete imaging in the wound, especially the dermal margins, without missing any areas.

Along with a smaller device and an automated approach, video mosaicking (converting videos into mosaics) will be a useful related advance to display larger areas of tissue. Video capture with the handheld confocal microscope (Vivascope 3000) allowed the user to rapidly access large areas in wounds (and entire areas in smaller wounds), including the entire epidermal margin and deeper dermal margins. The video acquisition procedure provides the flexibility for the imaging to be adapted to the arbitrary topography of the wound. However, discontinuities or "jumps" in the videos due to sudden microscopic topographical changes on the wound surface occurred, along with blurring and artifacts (dark or saturated areas), all of which must be processed by partitioning the videos into subvideos to create submosaics. This partitioning is currently done manually and consumes considerable time, therefore, more work on the automation of the video-mosaicking approach is required. Development of an automated approach is in progress, with initial demonstrated capability for imaging margins of BCC lesions. $^{24}$

For all the lesions, our clinicians (coauthors CSJC, MC, and OY) found that images, videos, and video mosaics exhibited overall clinically acceptable quality with regard to resolution, contrast, and appearance of features postablation. Identification of the epidermal, peripheral, and deep dermal margins was feasible due to the immediate recognition of relevant features specific to each region. Furthermore, the presence of artifacts (air bubbles, saturation) was accurately identified in the image and video assessments of each margin. Saturation in the images and videos appeared to be due to dryness of the postablated tissue that varied due to lesion location or patient characteristics. Thus, our approach, with optimal choice of laser ablation parameters to control thermal coagulation and enable postablation RCM imaging with a contrast agent, appears to be inherently working, and we believe that it should be advanced toward larger clinical studies and testing. Of course, further development in instrumentation and methodology will be necessary to support further advances in the clinic. While aluminum chloride appears to work well, reflectance contrast may sometimes fail to detect small tumors that may look similar to dermal structures such as hair follicles. Surely, a more specific contrast agent that further enhances tumor-to-dermis contrast could help for differentiating small residual tumors from normal dermal structures.

\section{Disclosures}

Milind Rajadhyaksha is a former employee of and owns equity in Caliber Imaging and Diagnostics (formerly, Lucid Inc.), the company that manufactures and sells the VivaScope confocal microscope. The VivaScope is the commercial version of an original laboratory prototype that was developed by Rajadhyaksha when he was at Massachusetts General Hospital, Harvard Medical School. None for the other coauthors.

\section{Acknowledgments}

We thank the NIH for funding support (grant R01EB020029 from NIBIB's Image-guided Interventions program, and partially, MSKCC's Center Core grant P30CA008748). Dr. Yélamos thanks the Beca Excelencia Fundación Piel Sana. We are grateful to Mr. Steven Wilson and Mr. Reza Afzalneia for preparing frozen histology and Ms. Christine Chang for her help with the patient consents. Finally, we thank Dr. Kivanc Kose for providing technical assistance with image stitching and producing videomosaics.

\section{References}

1. A. Lomas, J. Leonardi-Bee, and F. Bath-Hextall, "A systematic review of worldwide incidence of nonmelanoma skin cancer," Br. J. Dermatol. 166(5), 1069-1080 (2012).

2. C. Berking et al., "Basal cell carcinoma-treatments for the commonest skin cancer," Dtsch Arztebl Int. 111(22), 389-395 (2014). 
3. H. T Greenway et al., "Mohs microscopic surgery and cutaneous oncology," in Surgery of the Skin: Procedural Dermatology, J. K. Robinson et al., Eds., 3rd ed., Elsevier Saunders (2015).

4. G. Goldenberg and O. Hamid, "Nonsurgical treatment options for basal cell carcinoma-focus on advanced disease," J. Drugs Dermatol. 12(12), 1369-1378 (2013).

5. O. A. Ibrahimi et al., " $755 \mathrm{~nm}$ alexandrite laser for the reduction of tumor burden in basal cell nevus syndrome," Lasers Surg. Med. 43(2), 68-71 (2011).

6. H. Kavoussi and A. Ebrahimi, "Treatment and cosmetic outcome of superpulsed $\mathrm{CO}_{2}$ laser for basal cell carcinoma," Acta Dermatovenerol. Alp. Pannonica Adriat. 22(3), 57-61 (2013).

7. J. Del Pozo and L. Rosende, "Basal cell carcinoma. Treatment with carbon dioxide laser vaporization," Adv. Cancer Res. Treat. 2013, 1-11 (2013).

8. H. Kavoussi, A. Ebrahimi, and M. Rezaei, "Treatment and cosmetic outcome of superpulsed $\mathrm{CO}_{2}$ laser for basal cell carcinoma," Acta Dermatovenerol. Alp. Pannonica Adriat. 22, 1-5 (2013).

9. Z. Tannous, "Combined $585 \mathrm{~nm}$ pulsed-dye and 1,064 nm Nd:YAG lasers for the treatment of basal cell carcinoma," Lasers Surg. Med. 46(1), 1-7 (2014).

10. S. Iyer et al., "Treatment of basal cell carcinoma with the pulsed carbon dioxide laser: a retrospective analysis," Dermatol. Surg. 30(9), 12141218 (2004).

11. H. R. Jalian et al., "Combined $585 \mathrm{~nm}$ pulsed-dye and 1,064 nm Nd: YAG lasers for the treatment of basal cell carcinoma," Lasers Surg. Med. 46(1), 1-71 (2014).

12. B. T. Nguyen et al., "Treatment of superficial basal cell carcinoma and squamous cell carcinoma in situ on the trunk and extremities with ablative fractional laser-assisted delivery of topical fluorouracil," $\mathrm{J}$. Am. Acad. Dermatol. 72(3), 558-560 (2014).

13. A. E. Ortiz, R. R. Anderson, and M. M. Avram, " 1064 nm long-pulsed $\mathrm{Nd}$ :YAG laser treatment of basal cell carcinoma," Lasers Surg. Med. 47(2), 106-110 (2015).

14. M. U. Javed and M. Murison, "The combined $\mathrm{CO}_{2}$ laser and photodynamic therapy of multiple BCC's in a facial port wine stain," J. Plast Reconstr. Aesthetic Surg. 69(1), e10-e12 (2016).

15. R. Smucler et al., "Ultrasound guided ablative-laser assisted photodynamic therapy of basal cell carcinoma (US-aL-PDT)," Photomed. Laser Surg. 30(4), 200-205 (2012).

16. S. A. Fox et al., "Raman spectroscopy differentiates squamous cell carcinoma (SCC) from normal skin following treatment with a highpowered $\mathrm{CO}_{2}$ laser," Lasers Surg. Med. 46(10), 757-772 (2014).

17. C. S. Chen et al., "Confocal microscopy-guided laser ablation for superficial and early nodular Basal cell carcinoma: a promising surgical alternative for superficial skin cancers," JAMA Dermatol. 150(9), 994-998 (2014).

18. B. P. Hibler et al., "Carbon dioxide laser ablation of basal cell carcinoma with visual guidance by reflectance confocal microscopy: a proof-ofprinciple pilot study," Br. J. Dermatol. 174, 1359-1364 (2016).
19. H. Sierra et al., "Confocal microscopy to guide erbium:yttrium aluminum garnet laser ablation of basal cell carcinoma: an ex vivo feasibility study," J. Biomed. Opt. 18(9), 095001 (2013).

20. H. Sierra et al., "Confocal imaging-guided laser ablation of basal cell carcinomas: an ex vivo study," J. Invest. Dermatol. 135, 612-615 (2014).

21. Z. Tannous, A. Torres, and S. Gonzalez, "In vivo real-time confocal reflectance microscopy: a noninvasive guide for Mohs micrographic surgery facilitated by aluminum chloride, an excellent contrast enhancer," Dermatol. Surg. 29, 839-846 (2003).

22. M. Lukac, B. Majaron, and T. Rupnik, "Ablative and thermal effects of Er:YAG laser on human tissue," in Laser in der Medizin Laser in Medicine, W. Waidelich, R. Waidelich, and J. Waldschmidt, Eds., Springer, Berlin, Heidelberg (1998).

23. B. Larson et al., "Fundamentals and technology advances: fundamentals of reflectance confocal microscopy," Chapter 1 in Reflectance Confocal Microscopy of Cutaneous Tumors, 2nd Ed., S. G. Gonzalez et al., Eds., NovaTechset Publishers, Madrid (2017).

24. K. Kose et al., "Video-mosaicing of reflectance confocal images for examination of extended areas of skin in vivo," Br. J. Dermatol. 171(5), 1239-1241 (2014).

25. M. L. Marino et al., "Improving lesion localization when imaging with handheld reflectance confocal microscope," Skin Res. Technol. 22(4), 519-520 (2016).

26. E. S. Flores et al., "Intraoperative imaging during Mohs surgery with reflectance confocal microscopy: initial clinical experience," J. Biomed. Opt. 20(6), 061103 (2014).

27. A. Scope et al., "In vivo reflectance confocal microscopy of shave biopsy wounds: feasibility of intraoperative mapping of cancer margins," Br. J. Dermatol. 163(6), 1218-1228 (2010).

28. D. J. Rohrbach et al., "Preoperative mapping of nonmelanoma skin cancer using spatial frequency domain and ultrasound imaging," Acad. Radiol. 21(2), 263-270 (2014).

29. N. Iftimia et al., "Combined reflectance confocal microscopy-optical coherence tomography for delineation of basal cell carcinoma margins: an ex vivo study," J. Biomed. Opt. 21(1), 016006 (2016).

30. N. Iftimia et al., "Handheld optical coherence tomography-reflectance confocal microscopy probe for detection of basal cell carcinoma and delineation of margins," J. Biomed. Opt. 22(7), 076006 (2017).

Heidy Sierra is an assistant professor at the University of Puerto Rico Mayaguez, Mayaguez Puerto Rico. Her work at Memorial Sloan Kettering Cancer Center included image-guided therapy approaches for the treatment of skin cancer. Her current research interests include computational imaging, machine learning, confocal microscopy, phase microscopy, and multimodal imaging, all to be applied for guidance of diagnosis and therapy of diseases. She is a member of SPIE and a member of OSA. She is the author/coauthor of several peerreviewed publications and a book chapter.

Biographies for the other authors are not available. 